\title{
Efectos de la inseguridad Ciudadana en el bienestar de la población
}

Mg. Armando Leiva Tarazona

leivatarazona@hotmail.com

Dr. Alejandro Ramírez Ríos

aramirezr@ucv.edu.pe

Universidad Cesar Vallejo- Lima Perú

\section{RESUMEN}

En este artículo se hace una revisión de literatura del problema sobre la violencia e inseguridad ciudadana los cuales que causan una agresión física y psicológica de la población y su efecto trasciende negativamente en el bienestar y calidad de vida de las personas y el resultado de esta investigación que sirva de un reto a los gobernantes para plantear los objetivos viables para contrarrestar la delincuencia mejorando las políticas públicas de la seguridad ciudadana, se aplicó el método de análisis y síntesis como primer paso se observó los fenómenos de violencia e inseguridad ciudadana en sus dimensiones objetiva y subjetiva para descubrir las causas que originan dichos fenómenos y se demuestra que dichas causas originan efectos que disminuye el bienestar, calidad de vida y la felicidad de la población, por consiguiente el objetivo de esta investigación fue determinar los efectos de la violencia e inseguridad ciudadana en el bienestar de la población, se concluye que la violencia e inseguridad ciudadana en su dimensión objetiva causa daños físicos y económicos con un efecto del deterioro de la salud, económica y laboral de las personas que sufren directamente de estos hechos y en la dimensión subjetiva causa una percepción de inseguridad, miedo, temor y desconfianza que afecta la parte emocional, psicológico y cognitivo alterando la salud mental con cambios en sus actitudes, rutinas, hábitos e interrelaciones personales, comprendido los efectos de ambas dimensiones que merman la calidad de vida, bienestar y la felicidad de la población.

Palabras claves: Efectos, inseguridad ciudadana, bienestar. 


\title{
Effects of citizen insecurity on the well-being of the population
}

\begin{abstract}
ABATRAC
This article reviews the literature on the problem of violence and citizen insecurity, which cause physical and psychological aggression in the population and its effect negatively affects the well-being and quality of life of people and the result of this research that serves as a challenge to the rulers to propose viable objectives to counteract crime by improving public policies of citizen security, the method of analysis and synthesis was applied as a first step, the phenomena of violence and citizen insecurity were observed in their objective dimensions and subjective to discover the causes that originate these phenomena and it is shown that these causes originate effects that diminish the well-being, quality of life and happiness of the population, therefore the objective of this research was to determine the effects of violence and citizen insecurity in the well-being of the population, it is concluded that That violence and citizen insecurity in its objective dimension causes physical and economic damage with an effect of deterioration of the health, economic and employment of the people who suffer directly from these events and in the subjective dimension it causes a perception of insecurity, fear, fear and distrust that affects the emotional, psychological and cognitive part, altering mental health with changes in their attitudes, routines, habits and personal relationships, inclusion the effects of both dimensions that reduce the quality of life, well-being and happiness of the population
\end{abstract}

Keywords: Effects, citizen insecurity, well-being

Artículo recibido: 05 de Mayo 2021 Aceptado para publicación: 20 de Junio 2021 Correspondencia: leivatarazona@hotmail.com Conflictos de Interés: Ninguna que declarar 


\section{INTRODUCCIÓN.}

La violencia y la inseguridad ciudadana es un problema incontrolable que enfrenta la población y los gobernantes de todos los países del mundo, en sus diferentes grados de riesgo de victimización de robos, extorciones y secuestros y que es percibido por la sociedad con un sufrimiento directo e indirecto de estos hechos con una desconfianza de las acciones de sus instituciones, en ese sentido los sentimientos de inseguridad de miedo al crimen es una preocupación de la población y del gobierno (Méndez, Otero, Perret, 2020) por el aumento de los delitos que impactan en el deterioro de la salud mental de los ciudadanos (Ávila, Martínez, Vera, Bahena, Musitu 2016 prf. 8) complementado con la desconfianza muy alta de sus instituciones municipales. (e tal Ávila 2016 prf. 1)

En esta revisión de la literatura se describe las causas y efectos de la violencia e inseguridad ciudadana en el bienestar de la población y su estudio será indispensable para mejorar la seguridad ciudadana para su normal desenvolvimiento en el desarrollo de sus habitantes porque esta es un obstáculo que afecta el desarrollo sustentable de un país (Keerver, Castillo, 2018)

La inseguridad tiene sus dimensiones bien determinadas que son la objetiva y subjetiva, la primera se entiende como el sufrimiento directo del crimen y la segunda se entiende como la percepción de inseguridad, miedo, temor y desconfianza de las instituciones (Carrión 2007 pf. 1). En ese sentido para estudiar esta relación inversa del bienestar y la inseguridad ciudadana se realizará una revisión teórica de la Inseguridad, percepción y desconfianza. En ese sentido la percepción se define como un proceso inicial de la actividad del cerebro que regula la generación de los órganos sensoriales que son la visión y el tacto y se considera como la parte subjetiva que realiza una abstracción del mundo exterior de lo que se observa de la realidad de los hechos, esta actividad es muy fundamental porque de ella dependen las otras actividades psicológicas tales como el pensamiento, memoria, aprendizaje y otros (Oviedo 2004 prf. 5 al 10), la desconfianza se define por un lado como la incompatibilidad de los intereses de la población con el otro actor que no cumple las acciones propuestas y por otro lado de la falencia de conocimiento del actor que conlleva a la incertidumbre (Rodríguez, Pandal Shrum 2018 prf. 10) y la percepción de inseguridad ciudadana se define como el temor que sufren las personas de ser víctima del crimen directo e indirecto: y el resultado de ello produce 
el daño físico y psicológico de las personas afectando su bienestar individual, Salud mental, su felicidad y su calidad de vida, entendiéndole la calidad de vida de las personas no depende solamente de los factores económicos sino también complementa el sentimiento de bienestar individual que genera la felicidad (Manjarrés, Baca 2019 prfs. 6, 8,9). En ese sentido el objetivo de esta revisión de literatura es determinar los efectos de la inseguridad en el bienestar de la sociedad.

\section{MÉTODOS}

En este artículo se realizó una revisión de la literatura con el objetivo de sintetizar las bases epistemológicas de la merma del bienestar de la población frente a la violencia e inseguridad ciudadana para ello se utilizo el método de la teoría general de sistemas de análisis y síntesis funcional y estructural del fenómeno y la relación y conexión de sus elementos de la unidad o todo de la investigación de la violencia e inseguridad al crimen que afecta el bienestar y la calidad de vida de la sociedad(De la Peña Velásquez 2018 prf. 9) . Así mismo como indica la teoría de Newton el método de análisis y síntesis se da en dos partes primero la observación del fenómeno para descubrir las causas y el segundo demostrar que dichas causas originan el fenómeno estudiado(Molina 2016 pfs.4, 6), en tal sentido en esta investigación el fenómeno observado es la violencia y la inseguridad y las causas descubiertas es la percepción de inseguridad, miedo y desconfianza y estas causas serán estudiadas como originan el efecto de daños físicos y psicológicos en la merma del bienestar de la población que, por consiguiente el método de análisis se define como el descubrimiento de las causas que originan un fenómeno observado y la síntesis como la devolución del proceso que averiguo para demostrar que dichas causas, exactamente generan los fenómenos que queremos explicar.

Para la revisión de la literatura se utilizó las revistas indexadas en la base de datos de Scopus, Proquest y Scielo del periodo de los últimos 15 años con las palabras claves bienestar, efectos, percepción e inseguridad.

\section{RESULTADOS}

Mediante la revisión de la literatura en los artículos científicos se describen las causas de la violencia e inseguridad ciudadana (fenómeno estudiado) que afecta el bienestar de la población. 
Las causas y los efectos de la violencia por el consumo de alcohol, consumo y venta de drogas, y la victimización de robos y asaltos es un problema que afecta la salud pública, la violencia no solamente genera daños físicos sino también el deterioro de la salud mental con la disminución de la calidad de vida en el bienestar de la personas con un efecto negativo en su desarrollo normal con el cambio de los hábitos de rutinas diarias así cómo salir de casa, transito por lugares, merma de las relaciones sociales y protección del hogar (Martínez, Ávila, Vera, Rivera y Musitu 2016 capitulo introducción prfs. 2 y 3) Así mismo se diferencian claramente la victimización y la percepción de inseguridad y ambos son los factores determinantes de la calidad de vida de las personas, así como la victimización directa que sufren las personas de robos, extorciones, amenazas, secuestros y otros que genera un miedo al delito, así mismo la percepción de inseguridad entendido como sufrimiento de desprotección de las instituciones y por ende de la probabilidad de ser víctima también origina el miedo al delito y estas generan cambios de hábitos de interrelación personal, de rutinas de no salir de casa, transito de lugares públicos, adopción de la seguridad de los hogares siendo estas restricciones que afecta la salud mental y calidad de vida en el bienestar de las personas(Martínez, Ávila, Vera, Rivera y Musitu 2017 prfs. 1, 2). Los efectos de la inseguridad ciudadana que afecta la salud mental influyen en el freno del desarrollo económico y social de un país afectando la actitud emprendedora y la productividad en las empresas aumentando los costes y gastos y esto que genera el incremento de precios con un efecto indirecto de la disminución de la demanda el cual económicamente afecta el bienestar de la población, (Pizarro 2021 prf. 1)

Así mismo la percepción es el que modela el comportamiento de las personas por consiguiente ella depende de las condiciones de la realidad que vive especialmente de las necesidades y la supervivencia en situaciones conflictivas. En tal sentido el sujeto y el medio ambiente son variables recíprocas que cumplen como mediador de las enfermedades en la salud psicológica de las personas generando específicamente como la perdida de relaciones interpersonales por la desconfianza y la incertidumbre (Vuanello 2009 prfs. 15, 16 y 17).La seguridad es una necesidad básica para el desarrollo normal de las personas en tal sentido cuando las instituciones encargadas no satisfacen con la necesidad de este servicio originan en la población una victimización directa e indirecta de delitos con efectos de daños físicos, económicos por la sustracción 
de sus bienes, pérdida de tiempo laboral, gastos médicos y en paralelo esta origina una percepción de inseguridad, miedo al crimen complementado por otros factores como la información de los medios de comunicación y la desconfianza de las instituciones afectando la salud mental, disminución de la calidad de vida, la satisfacción y la felicidad de las personas.(Muratori y Zubieta 2016 prf. 1, 4 y 5), El bienestar de la población no necesariamente depende de la inseguridad del crimen sino también de la confianza de las instituciones y efectividad de las políticas públicas del Estado y de otras variables (Gálvez 2019 prf. 14).

En la realidad el incremento de la inseguridad genera la misma población por que no denuncian por el miedo y las autoridades que no hacen justicia, así por ejemplo que solo uno de 10 víctimas denuncian a las autoridades policiales de este hecho delictivo y 1 de 100 denunciados son sentenciados y esta injusticia de las autoridades generan una acelerada inseguridad de la victimización, percepción de inseguridad, y percepción de incapacidad de las instituciones que origina el miedo de las personas de ser víctimas de un riesgo real o imaginario y en este contexto la inseguridad, la percepción de inseguridad y desconfianza de las instituciones afecta la satisfacción con la vida, deterioro del capital social y la felicidad de las personas . Así mismo vulnera la salud mental y calidad de vida de las personas con una alteración en sus actividades, cambio de sus actitudes como usar joyas, salir de noche, de poseer de dinero en efectivo, tarjetas de crédito, restricciones de interrelaciónales, actividades deportivas y sociales. (Martínez, Carreón 2020 Prfs. 3, 5, 6, 7,)

Los efectos de inseguridad se da en cuatro dimensiones en el objetivo, subjetivo, socio geográfico y desorden social, la dimensión objetiva se entiende como la acción ilegal que infringe el delincuente en la agresión física contra el derecho de su propiedad, subjetivo se entiende como la agresión emocional que origina el miedo y temor al crimen, y agresión cognitivo una percepción del riesgo, dimensión socio geográfico las características físicas de los espacios públicos y finalmente la dimensión desorden social también denominado el comportamiento anti social por las drogadictos que afecta los signos visuales con el comportamiento ofensivo en los espacios públicos estas dimensiones antes mencionadas afectan la calidad de vida y el bienestar de la población ( Stefanizzi, Verdoline 2016 Prfs 5,6) también los fenómenos que origina la precepción de inseguridad son el crimen organizado, la violencia, condiciones socio económicas, 
la corrupción y la urbanización y esta última es la relación urbanización con el crimen organizada( Wladyka, David, yaworsky, William 2017 prf. 3 La salud mental de las personas están relacionada con el entorno ambiental dentro de ella está considerado el espacio público que vienen a ser las vías, parques, plazas y otras como un escenario donde se desenvuelven y se interrelacionan las personas y la seguridad está en la responsabilidad de las municipalidades, en tal sentido dichos espacios públicos si no presta seguridad origina una percepción de inseguridad, temor y miedo al crimen y la desconfianza de las instituciones Municipales que esta afecta la salud mental y por ende la disminución de bienestar de la población ).(Ovares , Quiroz 2015 prfs. 1, 4, 5, 6, 7 ),. también el factor que contribuye en la percepción de inseguridad de miedo y temor de la población es la información de la prensa como televisión, periódicos revistos y otros de los casos delictivos que suceden en la realidad. (Meghan, Downey, Sharece; Alex del Carmen; Dobbs, Rhonda 2017 prf. 2, 3)

Tabla de los resultados de efectos de inseguridad en el bienestar de la población

\begin{tabular}{|c|c|c|c|c|}
\hline \multirow[b]{3}{*}{$\begin{array}{l}\text { Martínez, } \\
\text { Ávila, Vera, } \\
\text { Rivera } \\
\text { y Musitu } \\
\text { (2016) }\end{array}$} & \multicolumn{2}{|c|}{ Dimensión Objetiva } & \multicolumn{2}{|c|}{ Dimensión Subjetiva } \\
\hline & Causas & Efectos & Causas & Efectos \\
\hline & $\begin{array}{l}\text { Agresión física y } \\
\text { Despojo de } \\
\text { sus bienes }\end{array}$ & $\begin{array}{l}\text { Deterioro de la } \\
\text { Salud física, } \\
\text { Gastos en cli- } \\
\text { Nicas y interru- } \\
\text { pcion de sus } \\
\text { Actividades } \\
\text { laborales }\end{array}$ & $\begin{array}{l}\text { Percepción de } \\
\text { Miedo y } \\
\text { desconfianza }\end{array}$ & $\begin{array}{l}\text { Salud mental: } \\
\text { Cambios de } \\
\text { Rutinas, hábitos de } \\
\text { como salir de casa, } \\
\text { Merma de interrala- } \\
\text { ciones personales }\end{array}$ \\
\hline $\begin{array}{l}\text { Pizarro } \\
(2021)\end{array}$ & & & $\begin{array}{l}\text { Percepción de } \\
\text { miedo }\end{array}$ & $\begin{array}{l}\text { Salud mental: } \\
\text { Disminución en la } \\
\text { la productividad }\end{array}$ \\
\hline $\begin{array}{l}\text { Vuanello } \\
\text { (2009) }\end{array}$ & & Salud física & $\begin{array}{l}\text { Percepción de } \\
\text { Miedo esta } \\
\text { Relacionado con } \\
\text { el medio } \\
\text { ambiente }\end{array}$ & $\begin{array}{l}\text { Salud psicológica } \\
\text { Merma de interrala- } \\
\text { Ciones personales }\end{array}$ \\
\hline $\begin{array}{l}\text { Muratori } \\
\text { y Zubieta } \\
(2016)\end{array}$ & $\begin{array}{l}\text { Daños físicos y } \\
\text { económicos }\end{array}$ & $\begin{array}{l}\text { Perdida de tiem- } \\
\text { po laboral, gas- } \\
\text { tos económicos }\end{array}$ & $\begin{array}{l}\text { Percepción de } \\
\text { Inseguridad y } \\
\text { miedo }\end{array}$ & Salud mental \\
\hline $\begin{array}{l}\text { Gálvez } \\
\text { (2019) }\end{array}$ & & & $\begin{array}{l}\text { Desconfianza de } \\
\text { las instituciones } \\
\text { y de las } \\
\text { las políticas } \\
\text { publicas }\end{array}$ & Salud mental: \\
\hline $\begin{array}{l}\text { Martínez, } \\
\text { Carreón } \\
(2020)\end{array}$ & & & $\begin{array}{l}\text { Desconfianza de } \\
\text { Las instituciones }\end{array}$ & $\begin{array}{l}\text { Salud mental } \\
\text { Calidad de vida, } \\
\text { Bienestar y felicidad }\end{array}$ \\
\hline
\end{tabular}




\begin{tabular}{|c|c|c|}
\hline $\begin{array}{l}\text { Stefanizzi, } \\
\text { Verdoline } \\
2016\end{array}$ & $\begin{array}{l}\text { Agresión emo- } \\
\text { Cional y cognitiva }\end{array}$ & Salud mental \\
\hline $\begin{array}{l}\text { Ovares , } \\
\text { Quiroz } \\
\text { (2015) }\end{array}$ & $\begin{array}{l}\text { Percepción de } \\
\text { Miedo esta } \\
\text { Relacionado con } \\
\text { el medio } \\
\text { ambiente y la } \\
\text { desconfianza } \\
\end{array}$ & Salud mental \\
\hline $\begin{array}{l}\text { Meghan, } \\
\text { Downey, } \\
\text { Sharece } \\
\text { Carmen; } \\
\text { Dobbs, } \\
\text { Rhonda }\end{array}$ & $\begin{array}{l}\text { Percepción de la } \\
\text { información de los } \\
\text { medios de comu- } \\
\text { nicacion }\end{array}$ & Salud mental \\
\hline
\end{tabular}

\section{DISCUSIÓN}

En la literatura se llegaron a los resultados de los efectos del fenómeno de inseguridad ciudadana en sus dimensiones objetivo y subjetivo como indica (Vuanello (2009) que la violencia en lo objetivo causa agresión física y despojo de bienes personales que afecta con la salud física, gastos económicos en la atención médica, y problemas laborales, en lo subjetivo causa percepción de miedo que afecta la salud psicológica con el cambio de hábitos y vulneración de interrelaciones personales, igualmente (Muratori y Zubieta 2016) indica mas que de la agresión física la percepción de inseguridad de miedo tiene más efecto porque afecta la salud mental, calidad de vida el bienestar y la felicidad y para (Gálvez 2019) al margen de la agresión física y percepción del miedo la violencia en lo subjetivo causa la desconfianza de las instrucciones y políticas públicas y esta afecta directamente en el bienestar mental de la población asi mismo (Martínez, Carreón 2020) coincide que la desconfianza de las instituciones afecta en la salud mental, calidad de vida, y felicidad pero para (Stefanizzi, Bertolini 2016) la violencia causa una agresión emocional y cognitiva que afecta su estilo de vida de la población y (Ovares Quiroz 2015) considera que la relación sujeto medio ambiente es la base en la manifestación conductual de las personas tal sentido que toda la percepción relacionada con el medio ambiente de inseguridad causa enfermedades mentales que afecta la salud física y económica de la población y finalmente para (Meghan, Downey, Sharece, Carmen; Dobbs, Rhonda 2017) al margen de la agresión física, lo que afecta más la parte subjetiva de la población es la información de la violencia por los medios de comunicación como la televisión, periódicos, revistas y otros 


\section{CONCLUSIÓN.}

Habiéndose estudiado y analizado en la literatura a los fenómenos violencia criminal y la inseguridad ciudadana en sus dimensiones objetiva y subjetiva: se concluye en la primera dimensión causa una agresión física y económica directamente a las personas afectando la salud física, la economía familiar, gastos médicos, incumplimiento en la actividades laborables y en lo subjetivo causa una percepción de miedo, temor y desconfianza de las instituciones, y políticas públicas y este ambiente de inseguridad relacionado con el sujeto al intermedio afecta directamente la parte psicológica, emocional y cognitiva del ser humano cambiando los hábitos ,rutinas, relaciones interpersonales, la productividad en las empresas y el normal desarrollo de la economía siendo los efectos de ambas dimensiones que vulnera el bienestar y la felicidad de la población

\section{REFERENCIA BIBLIOGRÁFICA}

Ávila, Martínez, Vera, Bahena, Musitu (2016) Victimización, percepción de inseguridad y cambios en las rutinas cotidianas en México https://www.scielo.br/pdf/rsp/v50/es_0034-8910-rsp-S151887872016050006098.pdf

Keever, Castillo (2018) Percepción de Urban Inseguridad y sus implicaciones para el desarrollo sostenible https://www.scopus.com/record/display.uri?eid=2-s2.085071575411\&origin=resultslist\&sort=plf-

$\& \mathrm{src}=\mathrm{s} \& \mathrm{nlo}=\& \mathrm{nlr}=\& \mathrm{nls}=\& \mathrm{sid}=\mathrm{a} 95 \mathrm{f0724c} 77 \mathrm{c} 310674 \mathrm{c} 35716 \mathrm{c} 302 \mathrm{fc} 61 \& \mathrm{sot}=\mathrm{b} \&$ $\mathrm{sdt}=\mathrm{b} \& \mathrm{sl}=47 \& \mathrm{~s}=\mathrm{TITLE}-\mathrm{ABS}-$

KEY\%28perception+of+citizen+insecurity\%29\&relpos=31\&citeCnt=0\&search Term $=$

Méndez Otero Perret, (2020) Inseguridad Percibida en los Barrios de Santiago de Chile: La Importancia del Bienestar Subjetivo* https://www.scielo.br/scielo.php?script=sci_arttext\&pid=S0011$\underline{2582020000100204 \& \text { lang=es }}$

Ávila, Martínez, Vera, Bahena, Musitu (2016) Perception de inseguridad y cambios en la rutina diaria de México https://www.scopus.com/record/display.uri?eid=2s2.0-85015387212\&origin=resultslist\&sort=plf$\mathrm{f} \& \mathrm{src}=\mathrm{s} \& \mathrm{nlo}=\& \mathrm{nlr}=\& \mathrm{nl} \mathrm{s}=\& \mathrm{sid}=\mathrm{b} 7 \mathrm{ca} 8 \mathrm{~d} 0 \mathrm{e} 8205 \mathrm{f} 96850 \mathrm{~d} 31 \mathrm{f} 94121 \mathrm{f} 7 \mathrm{~b} 95 \& \mathrm{sot}=\mathrm{b}$ 
$\& s d t=b \& s l=47 \& s=$ TITLE-ABS-

KEY\%28PERCEPTION+OF+CITIZEN+INSECURITY\%29\&relpos=43\&cite

$\mathrm{Cnt}=6 \&$ searchTerm $=$

Oviedo (2004) La definición del concepto de percepción en psicología con base en la

Teoría

Gestalt

http://www.scielo.org.co/scielo.php?script=sci_arttext\&pid=S0123-

885X2004000200010

Manjerres y Baca (2019) Victimización por crimen, percepción de seguridad y satisfacción con la vida en Colombia http://www.scielo.org.co/scielo.php?script=sci_arttext\&pid=S012459962019000200133\&lang=es

García (2012) La estructura de la percepción de la inseguridad pública http://www.scielo.org.pe/scielo.php?script=sci_arttext\&pid=S172948272012000100005

Rodríguez Pandal, Shrun (2018) Sobre la desconfianza en la ciencia y la política: el $\begin{array}{llll}\text { brote } & \text { de } & \text { en }\end{array}$ http://www.scielo.org.mx/scielo.php?script=sci_arttext\&pid=S0187-

01732019000100205

De la Peña, Velásquez (2018) Algunas reflexiones sobre la teoría general de sistemas y el enfoque sistémico en las investigaciones científicas http://www.scielo.sld.cu/scielo.php?script=sci_arttext\&pid=S0257$\underline{43142018000200003}$

Carrión (2007) percepción inseguridad ciudadana BFLACSO-CS15-01-Carrión.pdf (flacsoandes.edu.ec)

Repositorio Digital FLACSO Ecuador: Percepción inseguridad ciudadana (flacsoandes.edu.ec)

Molina (2016) El método de análisis y síntesis y el descubrimiento de Neptuno*http://www.scielo.org.co/pdf/ef/n55/0121-3628-ef-55-00030.pdf

Martínez, Ávila, Vera, Rivera y Musitu (2016) Satisfacción con la vida, victimización y percepción de inseguridad en Morelos, México http://www.scielo.org.mx/scielo.php?script=sci_arttext\&pid=S0036$36342016000100007 \&$ lang=es 
Martínez, Ávila, Vera, Rivera, Musitu, Montero (2017) Percepción de inseguridad, victimización y restricciones en la vida cotidiana en función del ciclo vital, en Morelos, México http://www.scielo.org.co/scielo.php?script=sci_arttext\&pid=S1794$31082017000300183 \&$ lang=es

Pizarro (2021) los efectos de la inseguridad ciudadana sobre el emprendimiento: un freno al desarrollo de Latinoamérica https://www.scopus.com/record/display.uri?eid=2-s2.085097820296\&origin $=$ resultslist\&sort $=\mathrm{r}-\mathrm{f} \& \mathrm{src}=\mathrm{s} \& \mathrm{mlteid}=2-\mathrm{s} 2.0-$ $85097841308 \&$ mlttype $=$ ref $\&$ mltall $=$ t\&imp $=t \& \operatorname{sid}=183 \mathrm{dea} 871 \mathrm{c} 606 \mathrm{c} 5 \mathrm{f} 5245 \mathrm{f} 8 \mathrm{c} 2$ a1d6527d\&sot=mlt\&sdt=mlt\&sessionsearchid=183dea871c606c5f5245f8c2a1d $6527 \mathrm{~d} \&$ relpos $=0 \&$ citecnt $=0$

Vuanello (2009) Inseguridad urbana y sus efectos: percepción de los jóvenes http://www.scielo.org.co/pdf/crim/v51n2/v51n2a03.pdf

Muratori, Zubieta, (2016) La inseguridad subjetiva como mediadora del bienestar social y clima emocional La inseguridad subjetiva como mediadora del bienestar social y clima emocional (scielo.org.ar)

Gálvez (2019) ¿Cuáles determinantes se relacionan con la percepción de inseguridad? Un análisis estadístico y espacial para la ciudad de Bogotá, D. C.¿Cuáles determinantes se relacionan con la percepción de inseguridad? Un análisis estadístico y espacial para la ciudad de Bogotá, D. C. (scielo.org.co)

Martínez Cerrón (2020) percepción de la inseguridad y bienestar subjetivo en México. una aproximación cualitativa1 https://search.proquest.com/docview/2486553922/E3EB2E0E67C34D41PQ/2?a ccountid $=37408$

Stefanizzi, Verdoline (2016) Bordered communities: the perception of insecurity in fve European cities https://search.proquest.com/docview/2102163661/C8AE2F078F8A42F2PQ/8?a ccountid $=37408$

Wladyka, David, Yaorsky William (2017)The Impact of Researchers' Perceptions of Insecurity and Organized Crime on Fieldwork in Central America and Mexico 
https://search.proquest.com/docview/1979468434/C8AE2F078F8A42F2PQ/10? accountid $=37408$

Hollis, Meghan E; Downey, Sharece; Alex del Carmen; Dobbs, Rhonda R. (2017) The relationship between media portrayals and crime: perceptions of fear of crime among citizen

https://search.proquest.com/docview/1870777330/C72C578219374F98PQ/209?account $\mathrm{id}=37408$ 